\title{
Influence of Carbon Catabolite Repression on the G1 Arrest of Saccharomyces cerevisiae MATa Cells by $\alpha$ Factor
}

\author{
By TERESA RUÍZ, JULIO R. VILLANUEVA AND LUÍS RODRÍGUEZ* \\ Departamento de Microbiologia, Facultad de Biología, Universidad de Salamanca, Salamanca, \\ Spain
}

(Received 22 April 1983; revised 27 June 1983)

Cells of the yeast Saccharomyces cerevisiae of the a mating type were arrested at the G1 phase of the cell division cycle after treatment with $\alpha$ factor in a culture medium containing a high concentration $(2 \%, w / v$, or higher) of a catabolite-repressing sugar. In media containing either a lower concentration of sugar or a non-fermentable carbon source, the extent of Gl arrest induced by the pheromone was reduced or became undetectable. Under catabolite-derepressing conditions $\alpha$ factor was inactivated by a cells at a higher rate than that found in repressing media. These results indicate the existence of a close correlation between the action of $\alpha$ factor on a cells and conditions of catabolite repression or derepression. A joint mechanism of action of $\alpha$ factor and catabolite-repressing carbon sources on a cells is postulated.

\section{INTRODUCTION}

Heterothallic strains of Saccharomyces cerevisiae of the $\alpha$ mating type secrete into the medium a hormone-like substance, $\alpha$ factor, which induces in cells of the opposite mating type, a, a number of morphological and physiological changes presumably preparatory to conjugation (for a review, see Thorner, 1981). Such changes include the adoption of an aberrant, pear-like ('shmoo') morphology (Duntze et al., 1970) and a transient arrest at the initiation of DNA synthesis, with the subsequent accumulation of cells at the Gl phase of the cell cycle (BückingThrom et al., 1973).

Some changes induced in a cells by $\alpha$ factor are mimicked by a number of temperaturesensitive cell division cycle mutants when they are shifted to the restrictive temperature in glucose medium. Under these conditions, mutants cdc 28 , cdc 36 , cdc 37 and cdc 39 undergo G1 arrest, exhibit the shmoo morphology and have the ability to mate (Reed, 1980). Two of these mutations, cdc 36 and cdc 39, are also involved in the regulation of conjugation, since they can suppress a mutation in the STE 5 locus (Shuster, 1982 $a$ ) which confers to a cells resistance to the action of $\alpha$ factor (Hartwell, 1980). It has been recently shown that mutations cdc 28 , cdc 36 and cdc 39 are suppressed when mutants are grown in a carbon catabolite derepressing medium (Shuster, 1982b).

On the basis of the above mentioned data, we decided to investigate the possible relationship between the action of $\alpha$ factor on a cells and carbon catabolite repression. In this paper we report the existence of a close correlation between the extent of G1 arrest induced by $\alpha$ factor and the concentration of catabolite-repressing carbon source in the culture medium. We show that the shortening of G1 arrest under derepressing conditions is due, at least in part, to a faster inactivation of $\alpha$ factor.

\section{METHODS}

Strains and growth conditions. The standard haploid wild-type strain of Saccharomyces cerevisiae X 2180-1A (MATa) was obtained from the Yeast Genetics Stock Center, Donner Laboratory, Berkeley, Calif., USA. Cells were grown in a minimal medium containing $0.67 \%(w / v)$ yeast nitrogen base (without amino acids) (Difco) and a 
suitable carbon source, at the concentration $(\%, w / v)$ indicated in the Results section, at $30^{\circ} \mathrm{C}$, in a gyratory shaker at 200 r.p.m.

Treatment with $\alpha$ factor. Early-exponential phase cultures $\left(A_{600}=0 \cdot 20-0.25\right)$ were spun down and the cells resuspended in fresh medium at a density of 5-10 $\times 10^{6} \mathrm{cells}^{-1}$. $\alpha$ Factor was added to a final concentration of $1.25 \mu \mathrm{g} \mathrm{ml}^{-1}$ and the suspensions were incubated, at $30^{\circ} \mathrm{C}$, in a New Brunswick gyratory water bath shaker, model G76, at 300 r.p.m. Cell number was determined microscopically in a Thoma haemocytometer slide.

Invertase assay and glucose determination. Invertase was assayed at $30^{\circ} \mathrm{C}$, as described by Goldstein \& Lampen (1975). Glucose was determined by the same method except that the first part of the assay (incubation of samples with sucrose) was omitted.

Chemicals. Synthetic $\alpha$ factor was obtained from Peninsula Laboratories (San Carlos, Calif., USA). $N-\alpha-p$-TosylL-lysine chloromethyl ketone (TLCK), glucose oxidase and peroxidase were from Sigma. All other reagents were of analytical grade.

\section{RESULTS}

\section{Effect of $\alpha$ factor on $\mathrm{Gl}$ arrest under catabolite repressing and derepressing conditions}

In order to investigate the possible relationship between the action of $\alpha$ factor on a cells and catabolite repression, we studied the extent of G1 arrest of a cells induced by $\alpha$ factor in media containing different concentrations of carbon source.

At a glucose concentration of $2 \%$ the amount of $\alpha$ factor added was able to arrest growth of a cells for a period of about $3 \mathrm{~h}$ (Fig. 1). At glucose concentrations lower than $2 \%$, the time during which growth of a cells was maintained arrested by $\alpha$ factor decreased progressively. Glucose concentrations greater than $2 \%$ did not allow $\alpha$ factor to arrest growth of a cells for a period of time longer than the one found at $2 \%$ glucose (data not shown). Significant variations in the growth arrest times were not observed when these experiments were repeated four times.

Invertase production was followed as a marker for conditions of catabolite repression, since synthesis of that enzyme is known to be controlled by this regulatory mechanism (Dodyk \& Rothstein, 1964). At a glucose concentration of $0.5 \%$, or higher, invertase synthesis was repressed, while when the concentration of glucose was $0.1 \%$, or lower, synthesis of the enzyme was derepressed. Since at $0.5 \%$ glucose the extent of the arrest of a cell growth by $\alpha$ factor was shorter than that found at $2 \%$ glucose, and invertase synthesis was maintained repressed, we considered the possibility that the effect of $\alpha$ factor on a cells might be dependent on the concentration of glucose, per se, and not on conditions of carbon catabolite repression. To check this possibility we incubated a cells with $\alpha$ factor in the presence of other carbon sources. The results are shown in Fig. 2. When fructose (another catabolite-repressing sugar) was used at a concentration of $2 \%$ the effect of $\alpha$ factor was similar to that found with $2 \%$ glucose and invertase synthesis was repressed, while at $0.1 \%$ fructose growth of a cells was arrested for a shorter time

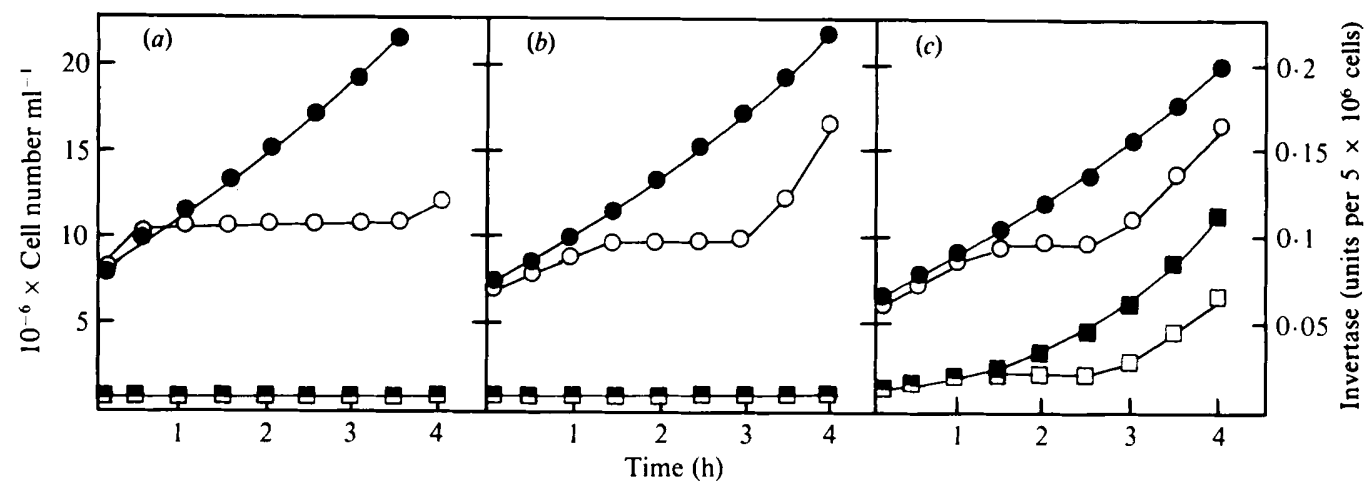

Fig. 1. Influence of glucose concentration on the action of $\alpha$ factor on a cells. Yeast cells of the a mating type were treated with $\alpha$ factor, as described in Methods, in YNB medium containing, $2 \%(a), 0.5 \%(b)$, or $0.1 \%(c)$ glucose. Samples were taken and cell number and invertase activity were determined. Cell number ( $O$ ) and invertase activity $(\square)$ of control cultures untreated with $\alpha$ factor; cell number $(O)$ and invertase activity $(\square)$ of cultures treated with $\alpha$ factor. 


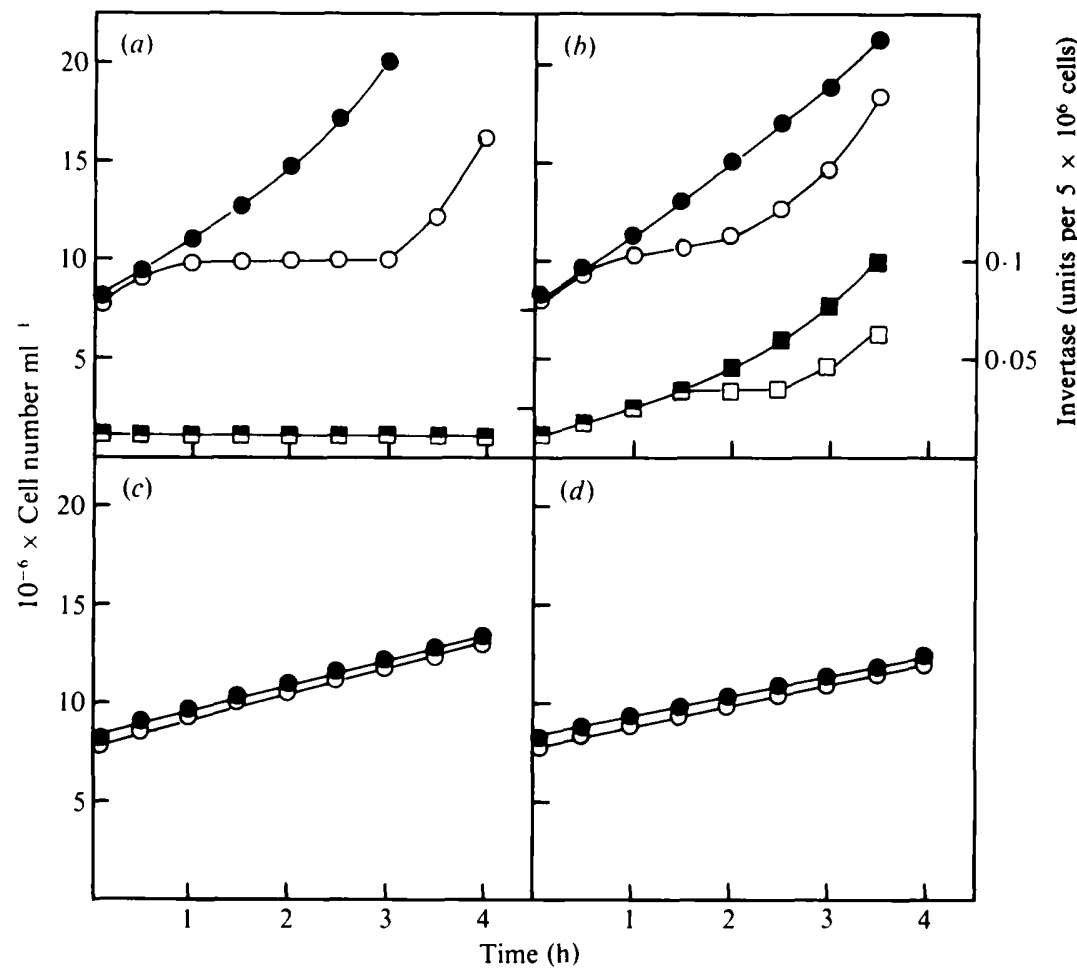

Fig. 2. Influence of carbon catabolite repressing and derepressing conditions on the action of $\alpha$ factor on a cells. Experimental conditions were those described in Fig. 1 except that a cells were grown in YNB medium containing $2 \%$ fructose ( $a$ and $b$ ), $2 \%$ glycerol $(c)$ or $2 \%$ pyruvate $(d)$ and the treatment with $\alpha$ factor was performed in YNB medium containing $2 \%$ fructose $(a), 0 \cdot 1 \%$ fructose $(b), 2 \%$ glycerol $(c)$ or $2 \%$ pyruvate $(d)$. Symbols are as in Fig. 1.

and invertase synthesis was derepressed. When incubation was carried out in a medium containing either pyruvate or glycerol (two non-catabolite-repressing carbon sources), at a concentration of $2 \%, \alpha$ factor did not arrest growth of a cells. Invertase synthesis was not followed in these media, as cells were fully derepressed after growing overnight under derepressing conditions.

Effect of catabolite repressing and derepressing conditions on the inactivation of $\alpha$ factor by a cells

The recovery from G1 arrest of a cells treated with $\alpha$ factor requires the inactivation of the pheromone (Chan, 1977), which seems to be due to its proteolytic digestion to biologically inactive fragments (Finkelstein \& Strausberg, 1979; Ciejek \& Thorner, 1979). In consequence, one of the possibilities that we considered to explain the different effect of $\alpha$ factor on the arrest of a cells grown under catabolite-repressing and derepressing conditions was that there might be a different rate of degradation of the pheromone by a cells. This possibility was tested by assaying the residual $\alpha$ factor that remained active after its incubation, for different times, with a cells in media containing different concentrations of glucose.

After the incubation in medium containing $2 \%$ glucose for $4 \mathrm{~h}$ all of the $\alpha$ factor used for the treatment of a cells was inactivated (Fig. 3). At incubation times of $3 \mathrm{~h}$ or less, in the same medium, some of the $\alpha$ factor remained active, since it was able to arrest the growth of fresh a cells in catabolite repressing medium, and the shorter the incubation, the longer the period of arrest. When the incubation of $\alpha$ factor with a cells was carried out in media containing lower concentrations of glucose there was a shortening in the times of arrest of the growth of fresh a cells by the residual $\alpha$ factor. Thus, for example, the $\alpha$ factor that remained active after 


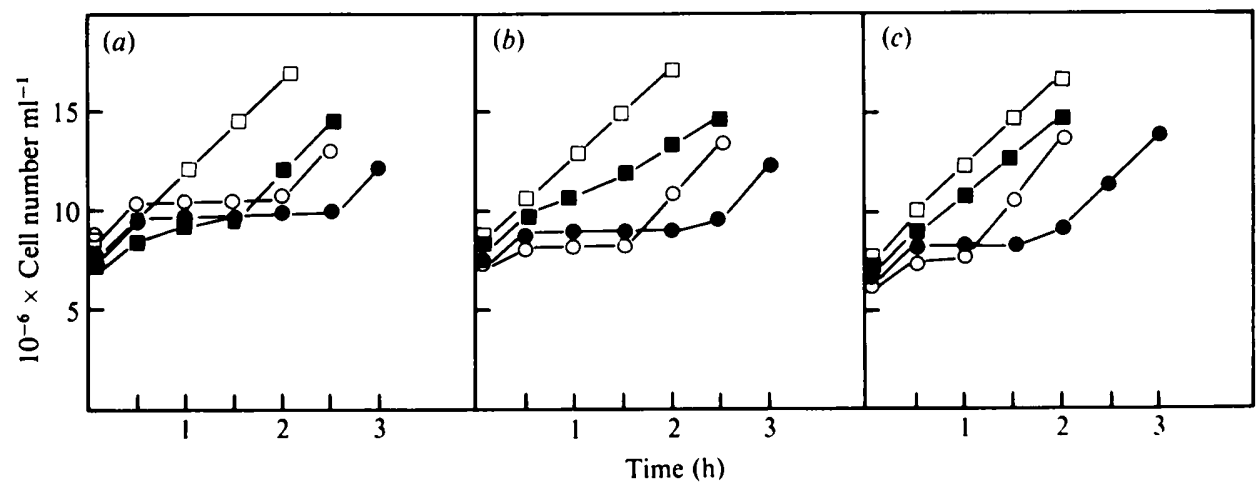

Fig. 3. Influence of carbon catabolite repressing and derepressing conditions on the inactivation of $\alpha$ factor. Yeast cells of the a mating type were treated with $\alpha$ factor, as described in Methods, in YNB medium containing $2 \%(a), 0 \cdot 1 \%(b)$ or $0.025 \%(c)$ glucose. Samples were taken at $1(O), 2(O), 3(\square)$ or $4(\square) \mathrm{h}$, the cells were removed by centrifugation and the supernatant fluids were supplemented with glucose to a final concentration of $2 \%$. Fresh a cells were resuspended in these supernatants at a final density of $7.5 \times 10^{6}$ cells $\mathrm{ml}^{-1}$ and incubated at $30^{\circ} \mathrm{C}$ with shaking. At the indicated times, samples were taken and the cell number was determined.

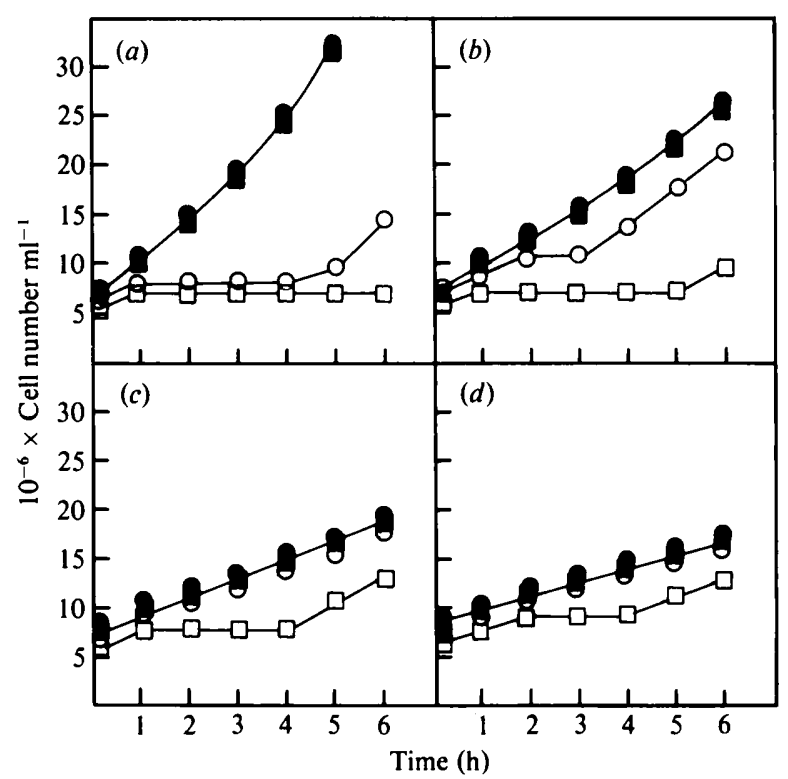

Fig. 4. Effect of TLCK on the inactivation of $\alpha$ factor by a cells. Yeast cells of the a mating type were grown in YNB medium containing $2 \%$ glucose $(a, b$ and $c)$ or $2 \%$ pyruvate $(d)$. After $14 \mathrm{~h}(a$ and $d)$ or when the glucose concentration fell to about $0.1 \%(b)$ or $0.025 \%(c)$, cells were harvested and treated with $\alpha$ factor as described in Methods, in YNB medium containing $2 \%(a), 0.1 \%(b)$ or $0.025 \%(c)$ glucose or $2 \%$ pyruvate $(d)$ in the absence or presence of TLCK (1 mM). At the indicated times, samples were taken and the cell number was determined. Cell number of control cultures untreated with $\alpha$ factor in the absence (O) or presence ( $\square$ ) of TLCK ; cell number of cultures treated with $\alpha$ factor in the absence $(O)$ or presence $(\square)$ of TLCK.

incubation in medium containing $0.025 \%$ glucose, for $2 \mathrm{~h}$, was only able to arrest the growth of fresh a cells for about $30 \mathrm{~min}$. This seems to confirm the hypothesis that the pheromone is more readily inactivated under catabolite conditions.

In order to test whether the inactivation of $\alpha$ factor was caused by a direct action of a cells the incubation of a cells with $\alpha$ factor was carried out in media containing different concentrations of glucose and in the presence of TLCK, a protease inhibitor, which has been reported to inhibit 
the surface-bound endopeptidase(s) involved in the proteolysis of $\alpha$ factor (Ciejek $\&$ Thorner, 1979) (Fig. $4 a-c$ ). A similar experiment was also performed in medium containing pyruvate as the only carbon source (Fig. $4 d$ ). Under both catabolite repressing and derepressing conditions the addition of TLCK to the incubation mixture lengthened the period of arrest of growth of a cells induced by $\alpha$ factor, and even under conditions where $\alpha$ factor does not normally halt the growth of a cells (media with $0.025 \%$ glucose or $2 \%$ pyruvate), the presence of TLCK enabled the $\alpha$ factor to arrest the division of a cells for several hours.

\section{DISCUSSION}

Results indicating that the action of $\alpha$ factor on a cells depends on the concentration of the carbon source in the incubation medium were reported by Lipke et al. (1976). However, a clear relationship between the response of a cells to $\alpha$ factor and catabolite repressing or derepressing conditions has not been previously established. From the results presented here it seems evident that the action of $\alpha$ factor on a cells is affected not only by the concentration but also by the nature of the carbon source present in the culture medium. Thus, in media containing either a non-fermentable carbon source or a fermentable sugar at low concentration (catabolite derepressing conditions) the time during which a cell growth is maintained arrested by $\alpha$ factor is undetectable or shorter than that found in media containing a fermentable sugar at a concentration high enough to produce catabolite repression.

Carbon catabolite derepression could produce a number of metabolic changes rendering the a cells less sensitive to the action of $\alpha$ factor. One of these changes seems to be an increased capacity of the a cells to inactivate the pheromone, presumably by proteolytic digestion mediated by surface-bound endopeptidase(s) (Ciejek \& Thorner, 1979). Results supporting this conclusion are the faster rate of $\alpha$ factor inactivation in catabolite-derepressing media and the fact that the addition of the protease inhibitor TLCK allows the arrest of a cell growth by $\alpha$ factor under catabolite derepressing conditions which normally prevent the action of the pheromone. The reason for this increase in the rate of degradation of $\alpha$ factor by a cells, in catabolite-derepressing media, could be: 1 , an activation of the peptidase(s) already present in the surface of a cells; 2 , an increase in the amount of surface bound peptidase(s) or 3 , an increased accessibility of the pheromone to the degradation system. Regarding this, it is of interest to mention that the different ability of a and $\alpha$ cells to degrade $\alpha$ factor seems to reside in the different capacity of their cell surfaces to exclude the pheromone (Thorner, 1981). In any case, the higher rate of degradation of $\alpha$ factor by a cells in catabolite-derepressing media might not be the only reason to explain the inability of the pheromone to arrest a cell growth since, for example, after an incubation in medium containing $0.025 \%$ glucose for 1 or $2 \mathrm{~h}$, enough active $\alpha$ factor still remains in the medium to halt the growth of fresh a cells under conditions of catabolite repression (Fig. 3 c).

The results presented here seem to confirm the existence of a common element linking the regulation of cell division, mediated by the mating factor, and the catabolite repression system, as has been suggested by Shuster $(1982 b)$. It may be speculated that the link between both mechanisms could be adenosine 3',5'monophosphate (cAMP) metabolism, as cAMP has been implicated in catabolite repression (Van Wijk \& Konijn, 1971; Sy \& Richter, 1972; Mahler \& Linn, 1978) and the changes induced in a cells by $\alpha$ factor seem to be mediated, at least in part, by a lowering of the intracellular cAMP level (Liao \& Thorner, 1980,1981). Since the action of $\alpha$ factor on a cells is prevented by catabolite derepression, we suggest that the morphological and physiological changes induced by the pheromone may be triggered by the reduction of the intracellular content of cAMP below a threshold level, carried out jointly by the mating factor and the catabolite-repressing carbon source; neither the pheromone nor the carbon source, acting separately from each other, would be able to reduce the cellular content of cAMP to a level low enough to initiate the cellular changes that are exhibited by a cells when they are treated with $\alpha$ factor under carbon catabolite-repressing conditions. In support of this putative cooperation of $\alpha$ factor and catabolite-repressing sugars in reducing the cellular content of cAMP is the fact that at $0.1 \%$ glucose or fructose the presence of $\alpha$ factor represses the synthesis of invertase (Figs $1 c$ and $2 b$ ). 
This work was supported in part by grant 0217/81 from Comisión Asesora de Investigación Cientifica y Técnica. We thank Mr N. Skinner for correcting the English version of the manuscript.

\section{REFERENCES}

BÜCKING-Throm, E., DUNTZE, W., HarTWell, L. H. \& MANNEY, T. R. (1973). Reversible arrest of haploid yeast cells at the initiation of DNA synthesis by a diffusible sex factor. Experimental Cell Research 76, 99-110.

Chan, R. K. (1977). Recovery of Saccharomyces cerevisiae mating-type a cells from $\mathrm{G} 1$ arrest by $\alpha$ factor. Journal of Bacteriology 130, 766-774.

CiejeK, E. \& ThORNER, J. (1979). Recovery of $S$. cerevisiae a cells from $G 1$ arrest by $\alpha$ factor pheromone requires endopeptidase action. Cell 18, 623-635.

DoDYK, F. \& RothsteIN, A. (1964). Factors influencing the appearance of invertase in Saccharomyces cerevisiae. Archives of Biochemistry and Biophysics 104, 478-486.

Duntze, W., MacKay, V. \& ManNey, T. R. (1970). Saccharomyces cerevisiae: a diffusible sex factor. Science 168, 1472-1473.

Finkelstein, D. B. \& Strausberg, S. (1979). Metabolism of $\alpha$ factor by a mating type cells of Saccharomyces cerevisiae. Journal of Biological Chemistry 254, 796-803.

Goldstein, A. \& LAMPEN, J. O. (1975). B-Fructofuranoside fructohydrolase from yeast. Methods in Enzymology 42, 504-511.

HaRTWell, L. H. (1980). Mutants of Saccharomyces cerevisiae unresponsive to cell division control by polypeptide mating hormone. Journal of Cell Biology 85, 811-822.

LiAO, H. H. \& THORNER, J. (1980). Yeast mating pheromone $\alpha$ factor inhibits adenylate cyclase. Proceedings of the National Academy of Sciences of the United States of America 77, 1898-1902.

LIAO, H. H. \& THORNER, J. (1981). Adenosine 3',5'phosphate phosphodiesterase and pheromone re- sponse in the yeast Saccharomyces cerevisiae. Journal of Bacteriology 148, 919-925.

Lipke, P. N., Taylor, A. \& Ballou, C. E. (1976). Morphogenic effects of $\alpha$-factor on Saccharomyces cerevisiae a cells. Journal of Bacteriology 127, 610618.

MAHLER, H. R. \& LINN, C. C. (1978). Exogenous adenosine $3^{\prime}, 5^{\prime}$-monophosphate can release yeast from catabolite repression. Biochemical and Biophysical Research Communications 83, 1039-1047.

REED, S. I. (1980). The selection of Saccharomyces cerevisiae mutants defective in the start event of cell division. Genetics 95, 561-577.

SHUSTER, J. R. (1982a). Mating-defective ste mutations are suppressed by cell division cycle start mutations in Saccharomyces cerevisiae. Molecular and Cellular Biology 2, 1052-1063.

SHUSTER, J. R. (1982b). "Start" mutants of Saccharomyces cerevisiae are suppressed in carbon catabolitederepresssing medium. Journal of Bacteriology 151, 1059-1061.

Sy, J. \& Richter, D. (1972). Content of cyclic 3',5'adenosine monophosphate and adenylyl cyclase in yeast at various growth conditions. Biochemistry 11, 2788-2791.

THORNER, J. (1981). Pheromonal regulation of development in Saccharomyces cerevisiae. In The Molecular Biology of the yeast Saccharomyces. Life cycle and inheritance, pp. 143-180. Edited by J. N. Strathern, E. W. Jones \& J. R. Broach. Cold Spring Harbor, New York: Cold Spring Harbor Laboratory.

VAN WiJK, R. \& KonIJN, T. M. (1971). Cyclic 3',5'AMP in Saccharomyces carlsbergensis under various conditions of catabolite repression. FEBS Letters 13, 184-186. 